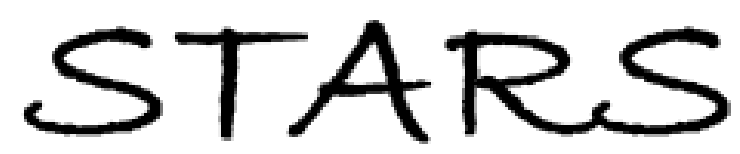

University of Central Florida

STARS

$1-1-2010$

\title{
Structural morphology of acoustically levitated and heated nanosilica droplet
}

\author{
Ranganathan Kumar \\ University of Central Florida \\ Erick Tijerino \\ University of Central Florida \\ Abhishek Saha \\ University of Central Florida \\ Saptarshi Basu
}

Find similar works at: https://stars.library.ucf.edu/facultybib2010

University of Central Florida Libraries http://library.ucf.edu

This Article is brought to you for free and open access by the Faculty Bibliography at STARS. It has been accepted for inclusion in Faculty Bibliography 2010 s by an authorized administrator of STARS. For more information, please contactSTARS@ucf.edu.

\section{Recommended Citation}

Kumar, Ranganathan; Tijerino, Erick; Saha, Abhishek; and Basu, Saptarshi, "Structural morphology of acoustically levitated and heated nanosilica droplet" (2010). Faculty Bibliography 2010s. 393.

https://stars.library.ucf.edu/facultybib2010/393

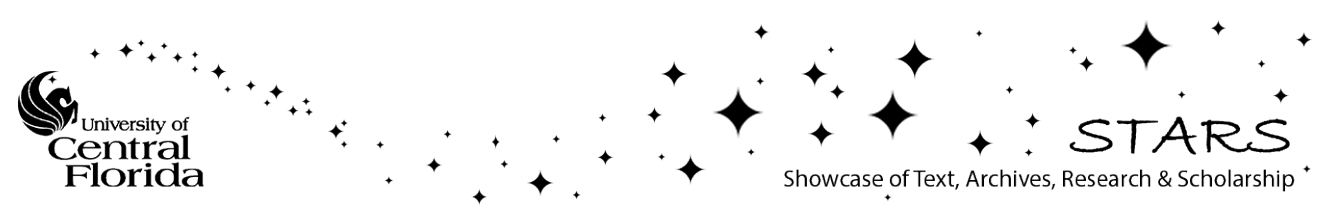




\section{Structural morphology of acoustically levitated and heated nanosilica droplet}

Cite as: Appl. Phys. Lett. 97, 123106 (2010); https://doi.org/10.1063/1.3493178

Submitted: 09 June 2010 . Accepted: 30 August 2010 . Published Online: 23 September 2010

Ranganathan Kumar, Erick Tijerino, Abhishek Saha, and Saptarshi Basu

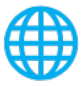

\section{ARTICLES YOU MAY BE INTERESTED IN}

Drying of acoustically levitated droplets of liquid-solid suspensions: Evaporation and crust formation

Physics of Fluids 14, 2289 (2002); https://doi.org/10.1063/1.1483308

Thermally induced secondary atomization of droplet in an acoustic field Applied Physics Letters 100, 054101 (2012); https://doi.org/10.1063/1.3680257

Scaling analysis: Equivalence of convective and radiative heating of levitated droplet Applied Physics Letters 100, 204104 (2012); https://doi.org/10.1063/1.4720092

\section{Applied Physics Reviews} Now accepting original research 


\title{
Structural morphology of acoustically levitated and heated nanosilica droplet
}

\author{
Ranganathan Kumar, ${ }^{1, a)}$ Erick Tijerino, ${ }^{1}$ Abhishek Saha, ${ }^{1}$ and Saptarshi Basu ${ }^{2}$ \\ ${ }^{1}$ Department of Mechanical, Materials and Aerospace Engineering, University of Central Florida, \\ Orlando, Florida 32816, USA \\ ${ }^{2}$ Department of Mechanical Engineering, Indian Institute of Science, 560012 Bangalore, India
}

(Received 9 June 2010; accepted 30 August 2010; published online 23 September 2010)

\begin{abstract}
We study the vaporization and precipitation dynamics of a nanosilica encapsulated water droplet by levitating it acoustically and heating it with a $\mathrm{CO}_{2}$ laser. For all concentrations, we observe three phases: solvent evaporation, surface agglomeration, and precipitation leading to bowl or ring shaped structures. At higher concentrations, ring reorientation and rotation are seen consistently. The surface temperature from an infrared camera is seen to be dependent on the final geometrical shape of the droplet and its rotation induced by the acoustic field of the levitator. With nonuniform particle distribution, these structures can experience rupture which modifies the droplet rotational speed.

(C) 2010 American Institute of Physics. [doi:10.1063/1.3493178]
\end{abstract}

Droplet evaporation has been studied extensively in the literature because of its wide applications in cloud physics, combustion of liquid fuel, ink-jet printing, thermal sprays, surface-patterning, and biological crystal growth. Majority of the fundamental studies have been done on drops on flat plates and surfaces. The solute particles in the drop move toward the edge of the drop in a well known "coffee ring" phenomenon 1,2 where the competing effects between the convection and Marangoni flows allow various deposit patterns. ${ }^{3}$ The ring that we report in this paper is a threedimensional toroidal structure from an acoustically levitated nanosilica spherical droplet. We explore solute transport and segregation in an evaporating levitated $500 \mu \mathrm{m}$ droplet at nanosilica concentrations of $0.1 \%-5 \%$ irradiated by a $\mathrm{CO}_{2}$ laser. As seen in the droplet evaporation studies on a solid surface, in a levitated droplet, the evaporation flux due to laser heating causes nonuniform temperature distribution. Irradiation increases surface temperature which sets up a surface tension gradient which induces Marangoni convection. Even if the Marangoni flow is expected to be weak due to its long time scale, solute segregation can occur near the edges. ${ }^{4}$ More importantly, the levitator acoustic field sets up a significant flow perturbation known as acoustic streaming. The time scale due to acoustic streaming is much shorter and we show that this effect is dominant in a levitated droplet which allows the formation of a bowl shaped drop at low concentrations and ring shape at high concentrations.

The containerless processing technique using levitation of magnetic, nonmagnetic materials and live animals have been shown to be effective in counteracting gravity. ${ }^{5-7}$ The drop shape depends on the balance between the curvatureinduced stress caused by the surface tension and the acoustic radiation pressure on the drop surface. ${ }^{7}$ The acoustic pressure on the droplet surface is such that the two polar regions contract inwards and the equatorial region expands outwards. However, a small droplet of $500 \mu \mathrm{m}$ used here does not deform into an oblate spheroid due to curvature effect.

A single axis $100 \mathrm{kHz}$ ultrasonic Tec5 levitator was used to suspend a $500 \mu \mathrm{m}( \pm 30 \mu \mathrm{m})$ nanofluid droplet contain-

${ }^{a)}$ Electronic mail: rnkumar@mail.ucf.edu. ing $10 \mathrm{~nm}$ silica particles in deionized water solution (0.1\%-5\% volume concentration). The suspended droplet was heated by a tunable infrared (IR) (10.6 $\mu \mathrm{m}$ wavelength) $\mathrm{CO}_{2}$ laser with a beam diameter of $2 \mathrm{~mm}$; the laser was tuned to $2.4 \mathrm{~W}$. The $\mathrm{CO}_{2}$ laser, a high speed camera and an IR camera were kept in the same horizontal plane and were synchronized using an external delay generator. The IRcamera was operated at $100 \mathrm{fps}$ with an integration time of $1.63 \mathrm{~ms}$ to interrogate the droplet surface temperature in the $20-80{ }^{\circ} \mathrm{C}$ range The change in temperature due to change in emissivity was found to be $0.03{ }^{\circ} \mathrm{C}$ and hence negligible. The high speed Fastec camera kept at $30^{\circ}$ to the laser axis recorded the heating event and subsequent diameter changes at $500 \mathrm{fps}$. The suspended droplet will remain at an antinode of the acoustic wave of the levitator. ${ }^{8}$ The velocity scale in the symmetric recirculation zone inside the droplet can be written as $u_{a} \sim\left(p_{a} / c^{2} \cdot \rho_{\text {air }}\right) f^{3 / 2} r^{2}\left(\mu_{\text {air }} / \mu_{\text {drop }}\right)\left(1 / \nu_{\text {air }}\right)^{1 / 2}$ $\left(u_{a}=\right.$ acoustic velocity; $p_{a}=$ antinode pressure; $f=$ resonant frequency of acoustic levitator; $c=$ speed of sound; $\nu$ $=$ kinematic viscosity; and $r=$ drop radius). When the resonant frequency increases, the velocity due to acoustic streaming is increased. For a frequency of $20 \mathrm{kHz}$, the velocity induced by acoustic streaming within the droplet was measured to be $10 \mathrm{~mm} / \mathrm{s}$ using particle image velocimetry ${ }^{9}$ in a $4 \mathrm{~mm}$ diameter droplet. A single recirculation cell was seen. The viscosity of $10 \mathrm{~nm}$ silica nanosuspension can increase $\mathrm{e}^{10,11}$ up to $40 \%$ at volume concentration of $2 \%$. At $0.5 \%$ concentration, dynamic light scattering technique and transmission electron microscope showed ${ }^{10}$ that the agglomeration of nanosilica particles increases the effective diameter to $20 \mathrm{~nm}$. The viscosity of the suspension also shows monotonic increase with magnetic field. ${ }^{12}$ Since $u_{a}$ is inversely proportional to dynamic viscosity, and viscosity increases both due to the presence of nanoparticles and increased concentration due to vaporization, the velocity of flow inside the droplet is diminished by about a third of its value for the pure water droplet of the same size levitated at the same acoustic frequency.

Relative to acoustic streaming, the velocity scale due to Marangoni convection inside the droplet is given by $u_{M}$ $\sim(\alpha / r) \mathrm{Ma} \sim(d \sigma / d T)\left(\Delta T / \mu_{\text {drop }}\right) . u_{m}=$ velocity due to Ma- 


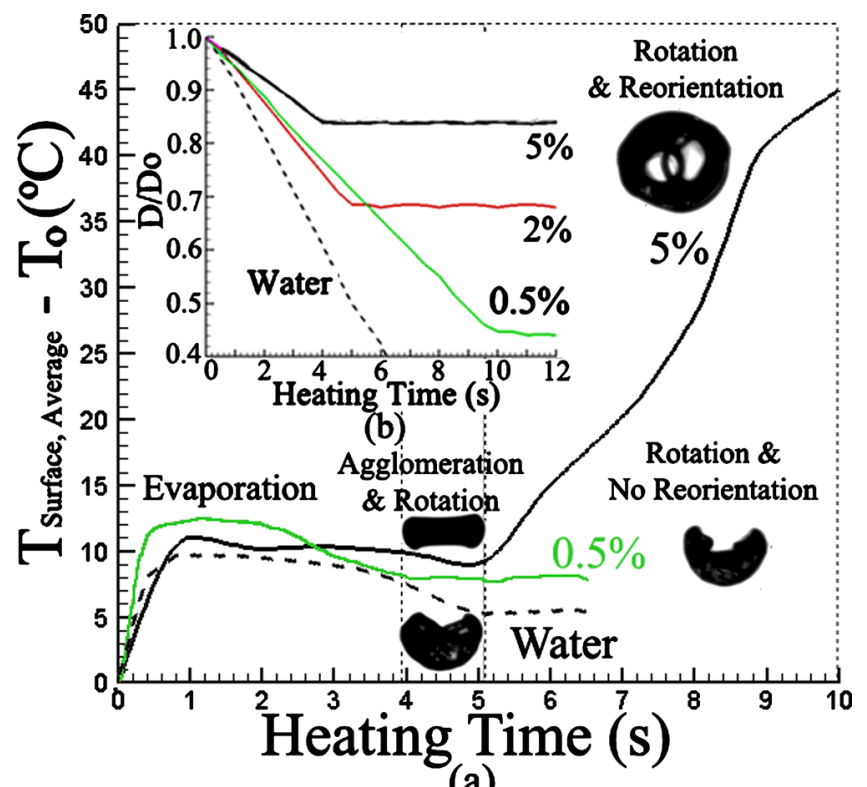

(a)

FIG. 1. (Color online) Average droplet surface temperature in time for water, $0.5 \%$ and $5 \%$ nanosilica volume concentration. Inset: diameter reduction in time for water, $0.5 \%, 2 \%$, and $5 \%$ concentration. (enhanced online). Video 3, [URL: http://dx.doi.org/10.1063/1.3493178.3].

rangoni flow; $\alpha=$ thermal diffusivity; $\mathrm{Ma}=$ Marangoni number; $d \sigma / d T$ is measured in our experiment as $-0.2 \mathrm{~N} / \mathrm{m}{ }^{\circ} \mathrm{C}$. A pendant droplet of $1 \mathrm{~mm}$ diameter with a $1{ }^{\circ} \mathrm{C}$ temperature gradient yields a velocity of $1 \mathrm{~mm} / \mathrm{s}$ from this analysis. For Ma $\sim 1000$, the velocity due to only Marangoni convection was measured ${ }^{13}$ to be $\sim 1 \mathrm{~mm} / \mathrm{s}$. This confirms that the Marangoni velocity scale, $u_{m}$, is an order of magnitude less than that due to acoustic streaming. Thus, despite the surface tension gradient at the surface, the solute transport inside the droplet, which is important to our observation and analysis, is predominantly due to acoustic streaming. This transport, however, is affected by the decrease in the droplet diameter due to surface vaporization.

Convective evaporation is generally characterized by $D^{2}$ law, with the droplet maintaining a spherical shape with time when no solute is present. In droplets containing nonevaporating phases like nanosilica, the vaporization rate decreases, in accordance with Raoult's law. ${ }^{14}$ The presence of solute results in a decrease in vapor pressure of water, which reduces surface vaporization. For the same heat flux, evaporation of pure water requires more latent heat reducing the available sensible heat for the temperature rise. This lowers the temperature rise for pure water (Fig. 1). Evaporation phase for water extends to $6 \mathrm{~s}$ as the drop diameter steadily decreases, while the diameter of a nanosilica droplet becomes a constant after sometime depending on the solute concentration. As the concentration is increased, the rate of decrease of diameter is lowered and became flat earlier.

When the nanosilica drop is heated radiatively, surface vaporization increases the solute concentration near the droplet surface in the absence of acoustic streaming. This increase in concentration is expected to be symmetric around the droplet surface for low radiation intensity. However, acoustic streaming coupled with radiative heating leads to a higher probability of concentration increase along the surface in the lower half of the droplet [Fig. 2(a)]. In addition, due to the pressure difference between the north and south poles,

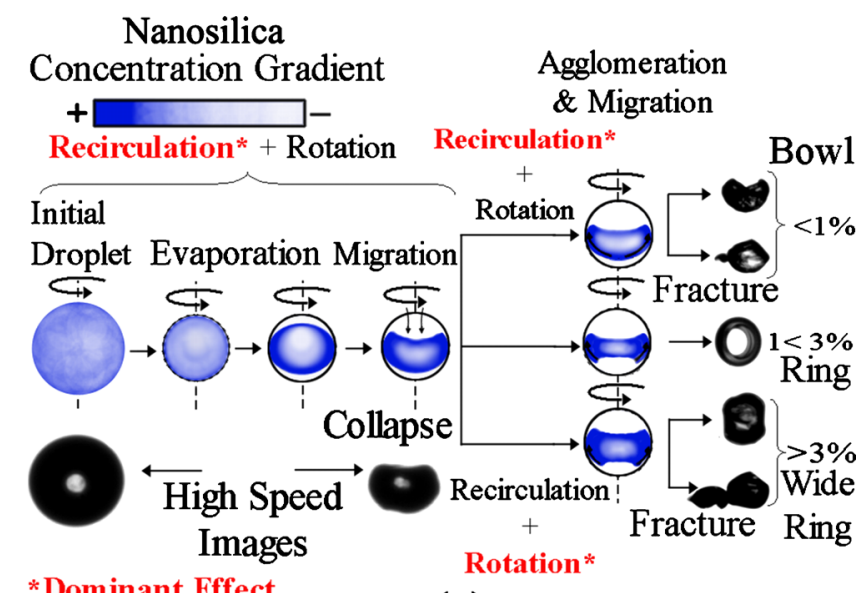

*Dominant Effect

(a)

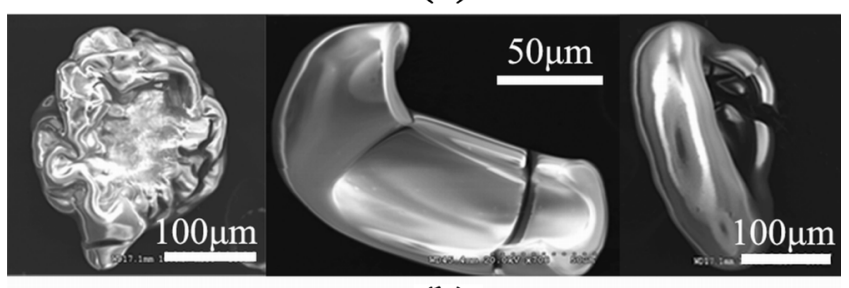

(b)

FIG. 2. (Color online) (a) Ring and bowl formation mechanisms. (b) SEM images of a bowl, a fractured ring, and a cracked ring, respectively. (enhanced online). Video 2, [URL: http://dx.doi.org/10.1063/1.3493178.2].

the particles tend to rotate about an axis tilted from the horizontal. This asymmetry in the flow also tends to accumulate the particles near the bottom of the droplet preferentially. Although the droplet does not rotate about the vertical axis initially, with increased rigidity in the lower half, the droplet starts rotating slowly about the vertical axis. Gravity plays a significant role indirectly in the phenomenon of caving in of the top half of the droplet. The presence of gravity leads to a downward shift from the pressure antinode, ${ }^{8}$ creating asymmetry between the top and bottom halves of the droplet. When the particles preferentially settle and agglomerate at the bottom of the droplet, the bottom part can withstand the acoustic force while the top part collapses to a new equilibrium position.

With time, the drop changes to a bowl structure from a predominantly spherical morphology [Fig. 2(b)]. It is expected that the nanosilica particles in the bowl shaped droplet agglomerates to form a weakly bonded shell type solid matrix. The formation of the solid structure leads to a sharp reduction in recirculation and rotation about the horizontal axis. The reduction in recirculation is marked by a sharp increase in rotational speed of the droplet about the z-axis due to the imposed acoustic force. Rotation introduces a large increase in recirculation about the equatorial plane as shown in Fig. 2.

This equatorial recirculation affects the droplet morphology depending on the concentration. Two distinct structures are observed: bowls form for $<1 \%$ concentration and rings form for $>2 \%$ concentration. For $<1 \%$ concentration, recirculation in the droplet is dominant due to lower initial viscosity over solid body rotation about the vertical axis. The time scale of agglomeration in this case is high, allowing for a predominant recirculation effect within the droplet. Rotational speed remains relatively low as the acoustic field in- 


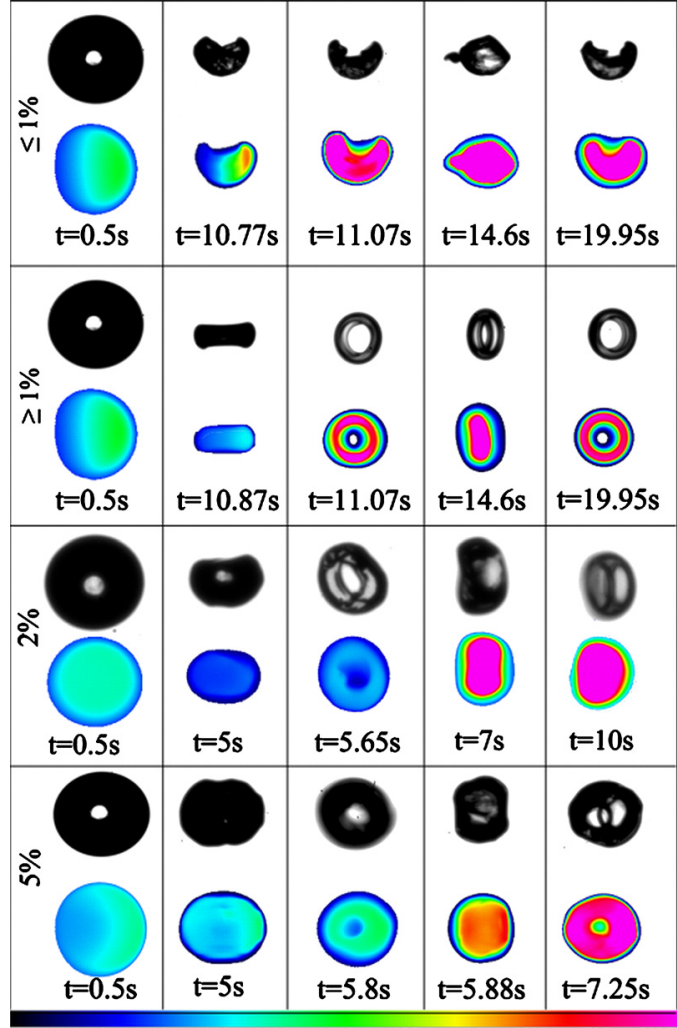

$24^{\circ} \mathrm{C} \quad 27^{\circ} \mathrm{C} 30^{\circ} \mathrm{C} \quad 33^{\circ} \mathrm{C} \quad 35^{\circ} \mathrm{C} \quad 37^{\circ} \mathrm{C} \quad 40^{\circ} \mathrm{C} \quad 43^{\circ} \mathrm{C} \quad 45^{\circ} \mathrm{C} \quad 47^{\circ} \mathrm{C} \quad 50^{\circ} \mathrm{C}$

FIG. 3. (Color online) High speed and IR images for various volume concentrations of silica nanoparticles. (enhanced online). Video 1, [URL: http://dx.doi.org/10.1063/1.3493178.1].

duces recirculation instead of rotation. Centrifugal force $\left(m_{p} \omega^{2} / r\right)$ acting on the particle mass, $m_{p}$, is low due to low rotational speed, $\omega$, which allows the particle to settle at the lower half of the droplet. Water content is ultimately depleted leaving behind a bowl shell structure.

However, if the initial nanosilica concentration is greater than $1 \%$, the agglomeration timescale is significantly reduced. With the help of the acoustic field, the particles that recirculate in a plane slightly tilted from the vertical axis induce a solid body rotation of the droplet at high rotational speeds, two orders of magnitude higher than at lower concentrations in some cases. This high rotation speed creates a high centrifugal force so that the particles migrate upwards and outwards in the equatorial plane. At this point, the droplet first deforms to an elliptical shape with its principal axis in the horizontal plane before forming a horizontal ring (Figs. 2 and 3 and Video 1, enhanced online). The ex situ analysis such as scanning electron microscopy (SEM) also confirms the final morphologies of the final precipitates to be ring and bowl shaped structures [Fig. 2(b)]. Both structures continue to rotate about the vertical axis due to the toroidal vortices around the levitated droplet called secondary acoustic streaming. ${ }^{15}$ The video clip shows that the bowl usually precedes the ring which is $\sim 300 \mu \mathrm{m}$ in diameter and $\sim 50 \mu \mathrm{m}$ in thickness. Higher concentration of particles $(\sim 3 \%)$ leads to wider rings in contrast to the thin rings observed at slightly over $1 \%$ concentration (Fig. 3 ).

At higher concentrations, another phenomenon observed consistently is the reorientation of the ring. When the con- centration is high, the timescale for agglomeration is lower, which leads to nonuniform particle distribution and reorients the ring to its stable vertical position. Note the difference in time for transition from horizontal to vertical ring for $1 \%$ (11 s) and 2\% (5.65 s) concentration. The IR images also show that temperature on the ring becomes high in a very short time in the vertical configurations at higher concentrations. After the structures are formed, there is negligible water content on the ring and hence most of the heat transferred is sensible heat rather than the latent heat. In this vertical position, the ring area exposed to radiative heat is larger than in the horizontal position as well as the area exposed by bowl shaped morphologies. Since radiative heat flux remains constant, a larger area exposed to heating translates to a sharp elevation in temperature (Fig. 1; see 5\% concentration).

Both ring and bowl shaped structures experience fracture in some cases [Figs. 2(a) and 3]. This is due to nonuniform particle deposition near the droplet surface. These nonuniformities form weak regions in the structure leading to rupture (Fig. 2). The broken parts are separated at first due to the centrifugal force induced by rotation, the acoustic field of the levitator forces the pieces back to the center of the levitator. Small parts of the structure can partially detach and form small flaps held by the acoustic field (see $\leq 1 \%$ concentration Fig. 2 and Video 2, enhanced online). The fractured pieces no longer fit perfectly and form a new structure (see 5\% concentration Fig. 1 and Video 3, enhanced online). The alteration of the structure shape interferes with the structural stability within the acoustic field and in some cases the rotational speed is increased. Droplet rotation about the levitator axis is unconstrained and any perturbation may lead to rotation. Our repeatable experiments show that this rotation is dependent on concentration.

In summary, a levitated nanosilica-filled drop undergoes various fascinating stages such as vaporization, precipitation before transitioning to a bowl or ring shape solid matrix. The rate at which the temperature rises depends on the structure geometry, position with respect to the heating source and speed of rotation induced by the acoustic field of the levitator.

${ }^{1}$ R. D. Deegan, O. Bakajin, T. F. Dupont, G. Huber, S. R. Nagel, and T. A. Witten, Nature (London) 389, 827 (1997).

${ }^{2}$ R. D. Deegan, O. Bakajin, T. F. Dupont, G. Huber, S. R. Nagel, and T. A. Witten, Phys. Rev. E 62, 756 (2000).

${ }^{3}$ J. Park and J. Moon, Langmuir 22, 3506 (2006)

${ }^{4}$ X. Xu, J. Luo, and D. Guo, Langmuir 26, 1918 (2010).

${ }^{5}$ E. H. Brandt, Nature (London) 413, 474 (2001).

${ }^{6}$ W. J. Xie, C. D. Cao, Y. J. Lu, Z. Y. Hong, and B. Wei, Appl. Phys. Lett. 89, 214102 (2006).

${ }^{7}$ W. J. Xie and B. Wei, Appl. Phys. Lett. 90, 204104 (2007).

${ }^{8}$ A. Y. Rednikov, H. Zhao, S. S. Sadhal, and E. H. Trinh, Q. J. Mech. Appl. Math. 59, 377 (2006).

${ }^{9}$ Y. Yamamoto, Y. Abe, Akiko Fujiwara, K. Hasegawa, and K. Aoki, Microgravity Sci. Technol. 20, 277 (2008).

${ }^{10}$ D. Milanova and R. Kumar, Appl. Phys. Lett. 87, 233107 (2005).

${ }^{11}$ D. Milanova and R. Kumar, J. Heat Transfer 130, 042401 (2008).

${ }^{12}$ P. D. Shima, J. Philip, and B. Raj, Appl. Phys. Lett. 95, 133112 (2009).

${ }^{13}$ J. J. Hegseth, N. Rashidnia, and A. Chai, Phys. Rev. E 54, 1640 (1996).

${ }^{14}$ S. Basu and B. M. Cetegen, J. Heat Transfer 130, 071501 (2008).

${ }^{15}$ A. Omrane, S. Santesson, M. Aldéna, and S. Nilsson, Lab Chip 4, 287 (2004). 